\title{
The significant global economic burden of bronchiectasis: a pending matter
}

\author{
Wei-jie Guan ${ }^{1,4}$, Xiao-rong Han ${ }^{1,4}$, David de la Rosa-Carrillo (1) ${ }^{2}$ and \\ Miguel Angel Martinez-Garcia ${ }^{3}$
}

Affiliations: 'State Key Laboratory of Respiratory Disease, National Clinical Research Center for Respiratory Disease, Guangzhou Institute of Respiratory Health, First Affiliated Hospital of Guangzhou Medical University, Guangzhou Medical University, Guangzhou, China. ${ }^{2}$ Pneumology Unit, Platon Hospital, Barcelona, Spain. ${ }^{3}$ Pneumology Dept, University and Politechnic La Fe Hospital, Valencia, Spain. ${ }^{4}$ Wei-jie Guan and Xiao-rong Han contributed equally.

Correspondence: Wei-jie Guan, State Key Laboratory of Respiratory Disease, National Clinical Research Center for Respiratory Disease, Guangzhou Institute of Respiratory Disease, First Affiliated Hospital of Guangzhou Medical University, 151 Yanjiang Road, Guangzhou, Guangdong, China. E-mail: battery203a163.com

@ERSpublications

Bronchiectasis confers a considerable economic burden on healthcare services worldwide http://ow.ly/ mTkF30nbEu7

Cite this article as: Guan W-J, Han X-R, de la Rosa-arrillo D, et al. The significant global economic burden of bronchiectasis: a pending matter. Eur Respir J 2019; 53: 1802392 [https://doi.org/10.1183/ 13993003.02392-2018].

Bronchiectasis should no longer be considered an "orphan lung disease" in view of its prevalence globally [1]. For instance, the prevalence of bronchiectasis has been found to range from 67 to 566.1 per 100000 inhabitants in Europe and North America (i.e. Germany [2], Spain [3], the UK [4] and the USA [5]), and has reached 1200 per 100000 inhabitants among those aged 40 years or older in China [6]. In fact, bronchiectasis is one of the three most common chronic airway inflammatory diseases (along with chronic obstructive pulmonary disease (COPD) and asthma) [7] which is increasingly gaining global awareness [8, 9]. Despite accumulating appreciation of the pathogenesis and the development of novel therapeutic interventions [10], the burden (particularly, economic) of bronchiectasis remains poorly understood compared with that of COPD and asthma.

Understanding disease burden is imperative for the improvement of the global management of bronchiectasis, particularly in geographical "hotspots". Several studies have documented the striking economic burden of bronchiectasis. In Germany, the mean age-standardised rate of bronchiectasis hospitalisations was 9.4 per 100000 inhabitants [11], which mirrored that in Spain (16.52-16.99 hospitalisations per 100000 inhabitants from 2004 to 2013) [12] and the USA (16.5 hospitalisations per 100 000 inhabitants) [13]. In the USA, bronchiectasis patients yielded significantly higher respiratory-related cost (mean USD 1607) than the matched controls [14]. More remarkably, the median inpatient cost accounted for USD 7827 in the USA [13], and the mean global cost was EUR 432.1 and EUR 4665.9 for emergency room visits and hospital stays in the wards (including transferrals and structural costs) in Spain, respectively [15]. Establishment of the diagnosis of Pseudomonas aeruginosa colonisation led to an $87 \%$ increase in the total healthcare cost (mean USD 67764 versus USD 36213) in the USA [16]. Importantly, greater bronchiectasis severity correlated with increased total expenditure, and most expenditure stemmed from in-hospital management with bronchodilators, inhaled steroids and inhaled antibiotics (particularly for severe exacerbation) [17]. These findings have provided crucial clues for the considerable economic burden of

Published online Feb 28, 2019; republished April 18, 2019 with a correction to the grant numbers listed in the author disclosures and support statement.

Received: Dec 172018 | Accepted: Dec 172018

Copyright OERS 2019 
bronchiectasis worldwide (table 1). However, previous studies have been limited by the relatively small sample sizes, a lack of matched controls (particularly those with concomitant COPD) and insufficient information regarding healthcare utilisation (i.e. use of medication).

Reassuringly, these limitations are now being addressed. In this issue of the European Respiratory Journal, DiEL et al. [18] have comprehensively evaluated the economic burden of bronchiectasis, based on a population of greater than 4800000 covered by German health insurance in 2012. There are important findings that should be highlighted. Per insured bronchiectasis patient, the total direct expenditure in 2012 (mean EUR 18634.57) was 30\% higher than that of the matched controls, and the cost of hospitalisation was $~ 55 \%$ higher in bronchiectasis patients than that in matched controls (mean EUR 6504.37 versus EUR 4183.65). In the USA, bronchiectasis patients yielded a mean of USD 2319 and USD 1607 for overall and respiratory-related costs in the first year after diagnosis [14]. Treatment is the core of bronchiectasis management, and hence, not surprisingly, constitutes an important aspect of the economic burden of bronchiectasis. The study by Diel et al. [18] revealed that outpatient drug costs (41.3\%), followed by hospitalisation costs $(34.9 \%)$, accounted most for the expenditure of bronchiectasis management. Of all medication classes, antibiotics (including intravenous and inhaled) and bronchodilators (i.e. salbutamol) accounted for most of the drug costs, because eradication of pathogenic bacteria (particularly, Pseudomonas aeruginosa) and airway clearance are two principal dogmas for bronchiectasis management. In concert with these findings, intravenous antibiotics and inhaled bronchodilators and steroids were the three major drug classes that accounted for most of the expenditure of both emergency room visits and in-hospital management in Spain [15, 17]. Inhaled bronchodilators, but not their combination with corticosteroids, accounted for a greater proportion of drug costs in Germany [18], which might be explained by the physician's preference for prescription. As expected, the economic burden of bronchiectasis extended well beyond conventional outpatient treatment and hospitalisation. For instance, the expenditure associated with the prolonged hospital stay was significantly greater than that of matched controls [18], which echoed the findings of the recent US study [16]. In addition, there remain considerable indirect costs for bronchiectasis management. Diel et al. [18] reported a noteworthy number of sick leave days (mean 40.5 days) and indirect cost (mean EUR 4230.49) associated with the loss of productivity [18]. These findings have important socioeconomic significance because such a loss of productivity may significantly reduce family income, which further predisposes to the limited access to healthcare services. We should again note that greater bronchiectasis severity correlated with significantly

TABLE 1 A summary of the current evidence regarding the global economic burden of bronchiectasis

\section{Direct expenditure}

- $31.0 \%$ higher than matched controls (mean EUR 18634.57 versus EUR 14 236.99) [18]

- USD 2319 and USD 1607 increase of overall and respiratory costs compared with matched controls [14]

Burden or expenditure for hospitalisation

- $56 \%$ higher hospital costs compared with matched controls (mean EUR 6504.37 in bronchiectasis) [18]

- 16.5 hospitalisations per 100000 inhabitants (higher in females) [13]

- 16.52-16.99 hospitalisations per 100000 inhabitants from 2004 to 2013 in Spain [12]

- 9.4 hospitalisations per 100000 inhabitants in the USA [11]

- Mean USD 7 respiratory-related incremental inpatient cost compared with matched controls [14]

- Mean cost of complementary explorations: EUR 147.1 for emergency room visits and EUR 331.8 for ward management [15]

- Mean global costs: EUR 432.1 for emergency room visits and EUR 4665.9 for hospital ward stay [15]

Expenditure for treatment prescription

- Mucoactive therapies: $~ 5$ times higher than matched controls (EUR 70.11 versus EUR 14.15) [18]

- Inhalation and home ventilation devices: $\sim 3$ times higher than matched controls [18]

- Antibiotics: significantly higher compared with matched controls (mean EUR 87728 versus EUR 56 219) [18]

- Mean annual cost per patient: EUR 2993, EUR 4732 and EUR 9999 for mild, moderate and severe bronchiectasis [17]

- Mean annual cost per patient: EUR 2041.9 and EUR 151.2 for inhaled and oral antibiotics, and EUR 759.9 for other inhaled therapy for clinically stable and exacerbation treatment [17]

\section{Miscellaneous}

- Similar sick leave (mean 40.5 versus 45.7 days) to matched controls [18]

- Mean sick leave: 13.4 (range 3-40) days in bronchiectasis patients [15]

- Similar productivity loss (mean EUR 4230.49 versus EUR 4776.50) to matched controls [18]

- Greater length of hospital stay (mean 19.6 versus 14.8 days) compared with matched controls [18]

- $5.8 \%$ higher annualised mortality compared to matched controls [18]

Shown are the four key aspects of the economic burden of bronchiectasis, including the direct expenditure, the cost or burden associated with hospitalisation for bronchiectasis, the expenditure for medication prescription, and other key aspects (i.e. mean sick leave days, length of hospital stay, loss of productivity). 
increased healthcare costs [17]. Hence, more emphasis should be given to the understanding of the increased impact of severe bronchiectasis on economic burden. Moreover, the prognosis of bronchiectasis might have been influenced by the presence of co-existing diseases that may elicit significantly greater economic burden. Indeed, bronchiectasis patients (particularly those with concomitant COPD) demonstrated a greater mortality rate compared with matched controls [18]. Similarly, a recent population-based study which recruited Medicare enrollees with prescription drug plans in the USA documented a notable proportion (51\%) of bronchiectasis patients with concomitant COPD who had frequent hospitalisation (due to respiratory tract infections) [19], which is known to be the risk factor of mortality. All the above-mentioned novel findings are expected to reshape our understandings of the underlying economic burden of bronchiectasis, which constitutes the cornerstone for advocating for a greater awareness of bronchiectasis healthcare worldwide.

The study, however, has some limitations that should be addressed by future investigations. For instance, the gender-adjusted mortality rate in girls aged under 15 years might have been underestimated. The difference of the prescription patterns between bronchiectasis patients and matched controls might have been diluted, given the fact that bronchiectasis frequently co-existed with COPD, which cannot be readily disentangled within the electronic database registry. More efforts are needed to systematically evaluate the post-discharge costs for bronchiectasis. Furthermore, it remains to be explored why mortality among patients with bronchiectasis without co-existing COPD was extremely low (which was significantly lower than that in controls) in the first year of follow-up.

There are some priorities and challenges for bronchiectasis research worldwide, particularly in developing countries. Incidence, prevalence and mortality remain sparsely documented in China [6], India [20] and Latin America, where the economic burden of bronchiectasis remains virtually unknown. Similarly, most economic analyses of the paediatric bronchiectasis population originated from developed, but not developing, countries [21]. Importantly, the economic burden of bronchiectasis might have been underreported in vast rural areas where recurrent respiratory tract infection and tuberculosis remain the dominant aetiologies of bronchiectasis [22]. However, the direct expenditure for bronchiectasis management might have been offset by the limited healthcare resources and relatively lower living standard in rural areas compared with urban regions. Given the significant economic burden of bronchiectasis compared with COPD, bronchiectasis should be incorporated into the list of lower respiratory tract diseases for the burden of respiratory disease report. Furthermore, establishment of a national bronchiectasis registry may facilitate assessment of how bronchiectasis contributes to the socioeconomic burden in developing countries. One way to mitigate the challenge of evaluating the economic burden of bronchiectasis in different countries and regions is the incorporation of data from the global burden of disease survey that would benefit from the collaboration with experts working in the field of epidemiology. Admittedly, population-based health insurance remains at its infancy in some developing countries, such as China; therefore, refinement of the health insurance system might provide more opportunities for further research, particularly for pilot investigations in major metropolitan areas.

Building on our expanded understanding, we have now obtained more solid evidence to justify the greater awareness of management and clinical research of bronchiectasis, which is indeed a common chronic airway inflammatory disease, aside from COPD and asthma [7]. An increase in investment in bronchiectasis research will ultimately help reduce medical expenditure via improved patient selection for interventions, thus saving more money [23]. Nonetheless, how best to identify the economic burden of bronchiectasis in developing countries where healthcare records are often incomplete or unavailable, still requires ongoing efforts.

Author contributions: W-J. Guan, X-R. Han, D. de la Rosa-Carillo and M.A. Martinez-Garcia drafted and critically reviewed the manuscript. All authors have approved the final submission.

Conflict of interest: W-J. Guan reports grants from National Natural Science Foundation (81870003), Pearl River S\&T Nova Program of Guangzhou (201710010097), Guangdong Province Universities and Colleges Pearl River Scholar Funded Scheme 2017 (2017), during the conduct of the study. X-R. Han has nothing to disclose. D. de la Rosa-Carillo has nothing to disclose. M.A. Martinez-Garcia has nothing to disclose.

Support statement: National Natural Science Foundation number 81870003, Pearl River S\&T Nova Program of Guangzhou number 201710010097 and Guangdong Province Universities and Colleges Pearl River Scholar Funded Scheme 2017 (to W-J. Guan). Funding information for this article has been deposited with the Crossref Funder Registry.

\section{References}

1 European Medicines Agency. Human Regulatory. Orphan Designation: Overview. www.ema.europa.eu/en/ human-regulatory/overview/orphan-designation-overview Date last updated: March 15, 2018. Date last accessed: December 14, 2018.

2 Ringshausen FC, de Roux A, Diel R, et al. Bronchiectasis in Germany: a population-based estimation of disease prevalence. Eur Respir J 2015; 46: 1805-1807. 
3 Monteagudo $\mathrm{M}$, Rodríguez-Blanco $\mathrm{T}$, Barrecheguren $\mathrm{M}$, et al. Prevalence and incidence of bronchiectasis in Catalonia, Spain: a population-based study. Respir Med 2016; 121: 26-31.

4 Quint JK, Millett ER, Joshi M, et al. Changes in the incidence, prevalence and mortality of bronchiectasis in the UK from 2004 to 2013: a population-based cohort study. Eur Respir J 2016; 47: 186-193.

5 Weycker D, Hansen GL, Seifer FD. Prevalence and incidence of noncystic fibrosis bronchiectasis among US adults in 2013. Chron Respir Dis 2017; 14: 377-384.

6 Lin JL, Xu JF, Qu JM. Bronchiectasis in China. Ann Am Thorac Soc 2016; 13: 609-616.

7 Martinez-Garcia MA, Polverino E, Aksamit T. Bronchiectasis and chronic airway disease. It is not just about asthma and COPD. Chest 2018; 154: 737-739.

8 Polverino E, Goeminne PC, McDonnell MJ, et al. European Respiratory Society guidelines for the management of adult bronchiectasis. Eur Respir J 2017; 50: 1700629.

9 Aliberti S, Masefield S, Polverino E, et al. Research priorities in bronchiectasis: a consensus statement from the EMBARC Clinical Research Collaboration. Eur Respir J 2016; 48: 632-647.

10 Flume PA, Chalmers JD, Olivier KN. Advances in bronchiectasis: endotyping, genetics, microbiome, and disease heterogeneity. Lancet 2018; 392: 880s-890s.

11 Ringshausen FC, de Roux A, Pletz MW, et al. Bronchiectasis-associated hospitalizations in Germany, 2005-2011: a population-based study of disease burden and trends. PLoS One 2013; 8: e71109.

12 Sánchez-Muñoz G, de Andrés AL, Jiménez-García R, et al. Time trends in hospital admissions for bronchiectasis: analysis of the Spanish national hospital discharge data (2004 to 2013). PLoS One 2016; 11: e0162282.

13 Seitz AE, Olivier KN, Steiner CA, et al. Trends and burden of bronchiectasis-associated hospitalizations in the United States, 1993-2006. Chest 2010; 138: 944-949.

14 Joish VN, Spilsbury-Cantalupo M, Operschall E, et al. Economic burden of non-cystic fibrosis bronchiectasis in the first year after diagnosis from a US health plan perspective. Appl Health Econ Health Policy 2013; 11: 299-304.

15 de la Rosa Carrillo D, Navarro Rolon A, Girón Moreno RM, et al. Cost of hospitalizations due to exacerbation in patients with non-cystic fibrosis bronchiectasis. Respiration 2018; 96: 406-416.

16 Blanchette CM, Noone JM, Stone G, et al. Healthcare cost and utilization before and after diagnosis of Pseudomonas aeruginosa among patients with non-cystic fibrosis bronchiectasis in the U.S. Med Sci (Basel) 2017; 5: E20.

17 de la Rosa D, Martínez-Garcia MA, Olveira C, et al. Annual direct medical costs of bronchiectasis treatment: impact of severity, exacerbations, chronic bronchial colonization and chronic obstructive pulmonary disease coexistence. Chron Respir Dis 2016; 13: 361-371.

18 Diel R, Chalmers JD, Rabe KF, et al. Economic burden of bronchiectasis in Germany. Eur Respir J 2019; 53: 1802033.

19 Henkle E, Chan B, Curtis JR, et al. Characteristics and health-care utilization history of patients with bronchiectasis in US medicare enrollees with prescription drug plans, 2006 to 2014. Chest 2018; 154: 1311-1320.

20 Natarajan S, Rudrawar P. Bronchiectasis in western India: clinical presentations and socio-economic burden. J Indian Med Assoc 2014; 112: 89-92.

21 Kapur N, Masters IB, Newcombe P, et al. The burden of disease in pediatric non-cystic fibrosis bronchiectasis. Chest 2012; 141: 1018-1024.

22 Gao YH, Guan WJ, Liu SX, et al. Aetiology of bronchiectasis in adults: a systematic literature review. Respirology 2016; 21: 1376-1383

23 Goeminne PC, Vanfleteren LEGW. Bronchiectasis economics: spend money to save money. Respiration 2018; 96: $399-402$. 